\title{
Development of a Hydraulic Energy Harvester for Shock Absorber
}

\author{
Longhan Xie*, Jiehong Li, Siqi Cai and Xiaodong Li \\ School of Mechanical and Automotive Engineering, South China University of Technology, Guangzhou, China \\ *Corresponding author
}

\begin{abstract}
This project is to design and manufacture a novel shock absorber with energy harvesting to reclaim vibration energy from automotive suspension system during driving. In order to improve safety and comfort, shock absorbers are used in automotive to remove vibration energy during driving, who adopt hydraulic system to convert vibration energy into heat so that vibration can be removed from the automotive suspension system. Obviously, the vibration energy is wasted. In this project, on the basis of satisfying the function request of automotive shock absorber, a mechanism for harvesting vibration energy is added to shock absorber to reclaim the vibration energy and convert it to electricity. Analysis shows that all four shock absorbers in one car can generate $2 \sim 4 \mathrm{~kW}$ electricity at $0.25 \sim 0.5 \mathrm{~m} / \mathrm{s}$ RMS suspension velocity, which can power all electronics in car and reduce automotive emission.
\end{abstract}

Keywords—shock absorber; energy harvesting; hydraulic

\section{INTRODUCTION}

Suspension is one of the most important parts of the vehicle, which connects the wheels to the car body. Recently, the research indicates that the suspension has a substantial influence on the fuel efficiency. Because of the roughness of the road, the vehicle will vibrate and this will not only lower the stability of the vehicle, but also waste the fuel. At this time, the conventional hydraulic shock absorber converts the vibration to heat, and dissipates it to air. Although this way can overcome the vibration rapidly, but the vibration energy is inevitably wasted. Due to there's a large quantity of vehicle in the world, we waste a huge amount of energy every day. In order to extract the vibration energy to drive the vehicle, the regenerative shock absorber has been suggested.

During the past decades, many researchers explored different principles and designs of regenerative shock absorber, which can be classified into two categories. The first category is based on conventional mechanism, which generate the power in hydraulic, pneumatic or magnetorheological way. Wang [1] et al proposed an idea of using the hydraulic motor to drive an electric generator in order to generate electric. Wendel [2] used a regenerative damp to extract the vibration energy of suspension. Noritsugu [3] investigated a pneumatic drive system, which store a part of the exhaust gas in order to reuse it in the air tank. Li and Tse[4]developed an energy-harvesting hydraulic damper to extract the vibration energy from suspension. Chen and Liao [5], as well as Sapinski [6]proposed to use MR damper to dismiss the vibration and extract the vibration energy. Beside the conventional mechanism, the second category is to generate the power in the electromagnetic way. When the coil and magnet assemblies move relatively with each other, electricity will be produced. There three configurations to produce electricity, including a linear electromagnetic generator[7,8,9], rack-pinion[10,11,12] and ball-screw[13,14,15]. Although the linear electromagnetic generator has a simple construction and works steadily, its efficiency is relatively low. The rack-pinion and ball-screw convert the up-and-down motion to rotation and have a very high efficiency, but both of them can not adapt the load with high frequency.

This paper aims at designing, characterizing a regenerative shock absorber based on hydraulic mechanism that can recover the vibration energy of the suspension. With a hydromotor, the shock absorber can utilize the hydraulic pressure to drive an electric generator in order to convert the vibration energy to electric energy. Using hydraulic mechanism not only can extract the vibration energy, but also be active controlled easily. For the hydraulic technique is mature enough, so the hydraulic energy harvester system is stable. This paper is organized as follow. In section2, we introduce the design of the energy harvester. In section3, we describe the system modeling and theoretical analysis. Then the conclusion is given in section4.

\section{DESIGN OF THE ENERGy HARVESTER}

In this section, we firstly describe the working principle of the active-control harvester. Then the test setup and experimental results of prototype will be given.

\section{A. Working Principle}

The harvester is shown as Figure I. This system can be clarified into two parts. The first part is a hydraulic damper, which connects the sprung mass to the wheel. The damper subassembly consists of two motion sensors, a spring, a piston, a cylinder and two valves. The damper is divided by the piston into two chambers. When the piston move up and down, it drives the hydraulic oil and convert the vibration energy to hydraulic energy. And due to the viscosity of hydraulic oil, vibration of the car body can be released. Besides the mechanism part, the second part is an energy harvester system. This energy recovery system consists of four check valves, a hydraulic motor, a generator with coupler, the active control system and an oil tank, etc. When the piston pushes the hydraulic oil, the oil can only pass through the check valve1 because the check valve1 and check valve4 are mounted relatively. The hydraulic oil with high kinetic energy will drive the hydraulic motor. Since the hydraulic motor and the electric generator are coupled by the coupler, the generator will rotate with the hydraulic motor and produce electricity. Then because 
after the oil has driven the generator, its pressure is less than the oil behind the valve4, so it can only pass through the check valve 2 and reach the oil tank. In this time, since the pressure of the tank's oil is lower than the oil in the left side of check valve3, it can not pass valves3. When the piston move up, the pressure of the oil in the left side of valve3 is lower than the pressure of the oil in the other side. Then the oil pass through the valve3 and drives the generator. It should be noted that the oil can not pass through the valve 2 for the same reason, but the oil can go through the valve4 and get back to the chamber of the damper.

The four check valves work as the rectifiers of the electric circuit, which convert the bidirectional flow to unidirectional flow. For the flow is unidirectional, the electric generator rotates unidirectional, too. In addition, the flow of the chamber is higher than the channel among the system, so it can drive the generator rotates quickly. When the hydraulic motor is rotating, we can change its rotating velocity by changing its flow displacement. But the active control is not discussed in this paper.

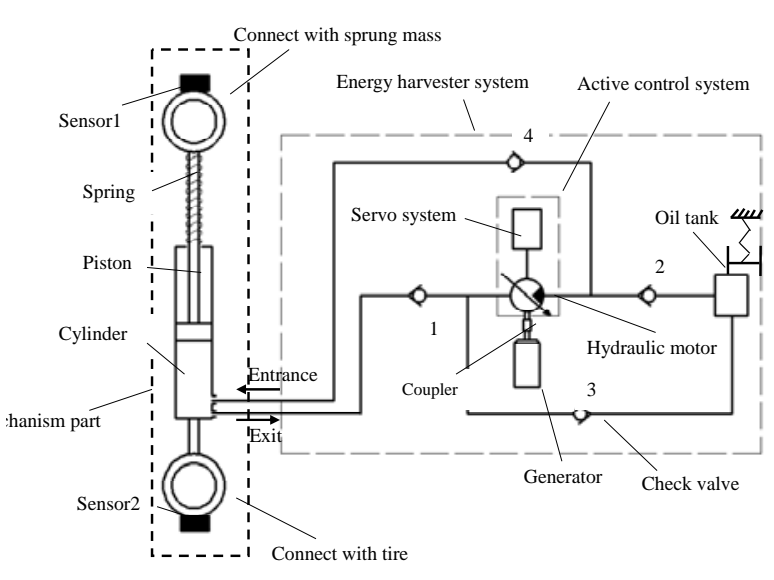

FIGURE I. THE SCHEMATIC OF THE HYDRAULIC ENERGY HARVESTER

\section{B. Prototype Device and Experimental Approach}

Following the working principle in section2.1, a model of prototype is developed. The schematic of the model is shown as Figure II, which consists of the hydraulic damper and the energy harvester system. The configuration of the damper is similar to the conventional damper, so it is easily to be mounted on the suspension. The damper and the spring are mounted in parallel. In addition, the configuration of energy harvester system is compact, so it is also easy to mount on the car body. The two systems are connected to each other by the pipeline. The prototype mounted on the vehicle is shown in the Figure III and Figure IV.

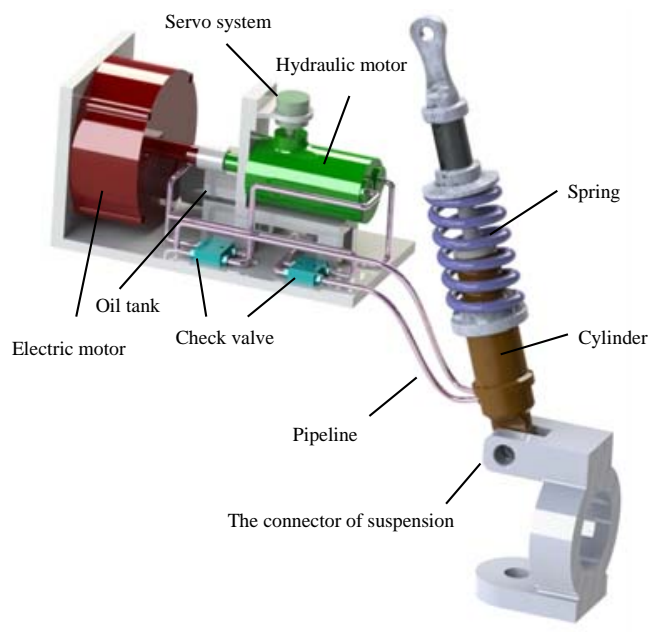

FIGURE II. THE MODEL OF THE HYDRAULIC ENERGY HARVESTER

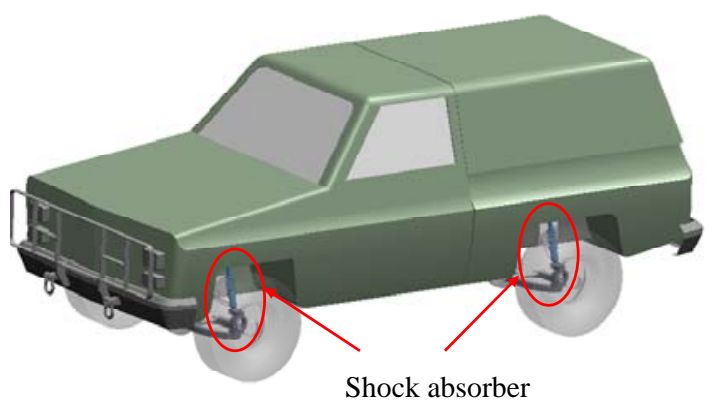

FIRGUR III. THE EQUIPMENT IN THE VEHICLE



FIGURE IV. THE PROTOTYPE MOUNTED ON THE SUSPENSION

\section{SySTEM MODELING AND THEORETICAL ANALYSIS}

When vehicles travel on the road, it will receive excitation from road roughness, baking forces etc., which discomfort the drivers. Shock absorbers can reduce the vibration by dissipating the vibration energy into heat waste. Although conventional damper can reduce the vibration effectively, its damping force is a constant. The conventional damper can be modelled as Figure $\mathrm{V}$ (a). $\mathrm{m} 1$ represents the unsprung mass and $\mathrm{m} 2$ represents sprung mass. The input of the system is the road roughness y0. This system has two degree of freedom, the movement of unsprung mass $\mathrm{y} 1$ and the movement of sprung mass $\mathrm{y} 2$. In addition, $\mathrm{k} 1$ is stiffness of the tire and $\mathrm{k} 2$ is stiffness 
of the suspension's spring. Moreover, C1 is a damping coefficient between the tire and the road, and $\mathrm{C} 1$ is considered to be a damping coefficient of the shock absorber. When the vehicle leaves the factory, C2 is set as a constant. So when the vehicle travels in different kind of road, its damping coefficient remains the same.

The model of hydraulic energy harvester is shown as Figure $\mathrm{V}$ (b), with a harvester system based on hydraulic damper. It's damping coefficient is C2. With the hydraulic energy harvester, the damper converts the vibration energy into electric energy. After the electricity is rectified by electric circuit, it can be stored in the battery.

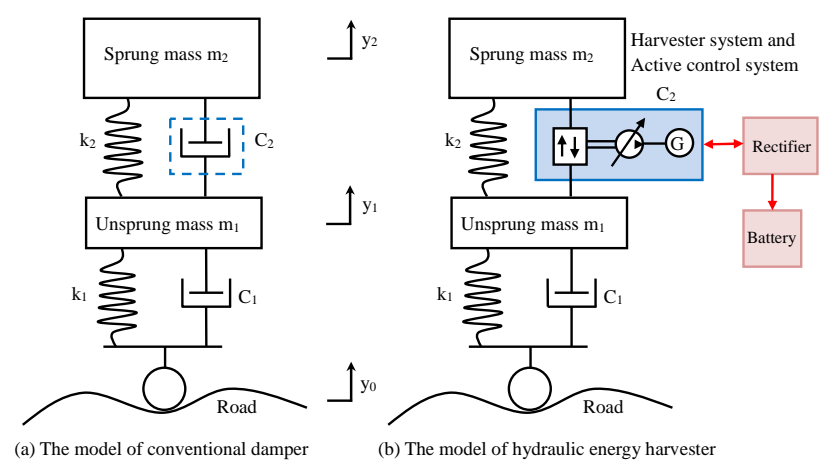

FIGURE V. QUARTER CAR MODEL

For this two-degree-freedom system of Figure V (b), we can get the following equation:



where the coefficients including of $\mathrm{m} 1, \mathrm{~m} 2, \mathrm{C} 1, \mathrm{k} 1$ and $\mathrm{k} 2$ are constant, only C2 is a variable. The coefficient y0 is the input of the system.

The relative displacement and velocity between the piston and the cylinder can be calculated by

$$
\left\{\begin{array}{l}
u=y_{2}-y_{1} \\
\stackrel{g}{u}=y_{2}-y_{1}^{g}
\end{array}\right.
$$

which are the relative displacement between unsprung mass and sprung mass.

The damping force can be produced following the relationship:

$$
F=C_{2} \stackrel{g}{u}
$$

The damping force is proportional to the relative velocity. The coefficient C2 is a variable which is controlled by changing the flow of the hydraulic damper. But the controlling method is not included in this paper.

With four check valves composing a rectifying system, the hydraulic oil drives the hydraulic motor unidirectionally. With the inner diameter $\mathrm{d}$ of the cylinder, efficiency $\eta_{1}$ and the displacement of hydraulic motor $\mathrm{V}$, the rotational speed of the motor can be calculated by equation(4).

$$
n=\frac{\pi d^{2}|u|}{4 V} \eta_{1} \times 60 \times 10^{6}
$$

$$
\begin{aligned}
& \text { With } a=\frac{\pi d^{2}}{4} \eta_{1} \times 60 \times 10^{6} \text {, the equation(4) can be } \\
& \text { produced as Eq.(5). }
\end{aligned}
$$

$$
n=\mathrm{a} \frac{|u|}{V}
$$

where $\mathrm{n}$ is proportional to $u$ and inversely proportional to $\mathrm{V}$. So the output power of the generator can be written as state equation:

$$
P=P_{0} \frac{a^{2}}{\mathrm{n}_{0}^{2}}\left(\frac{u}{V}\right)^{2}
$$

where $\mathrm{P}_{0}$ is the rated power of the generator and $\mathrm{n}_{0}$ is the rotational speed under the rated power. For both $\mathrm{P}_{0}$ and $\mathrm{n}_{0}$ are constant, the output power of the generator is proportional to the velocity of piston and inversely proportional to $\mathrm{V}$.

In the experimental test, we adopt a white noise as input of the harvester:

$$
y_{0}=-2 \pi f_{0} y_{0}+2 \pi \sqrt{G_{0} U_{0}} w
$$

where y0 is the excitation of the road, G0 is the coefficient of the road surface roughness, U0 is the velocity of vehicle, $\mathrm{w}$ is the Gaussian white noise with the mean value of zero, and f0 is lower cutoff frequency with the range from 0.06 to $0.07 \mathrm{~Hz}$. Figure VI shows the input of the road with surface of Rank $\mathrm{C}$ at $72 \mathrm{~km} / \mathrm{h}$. 


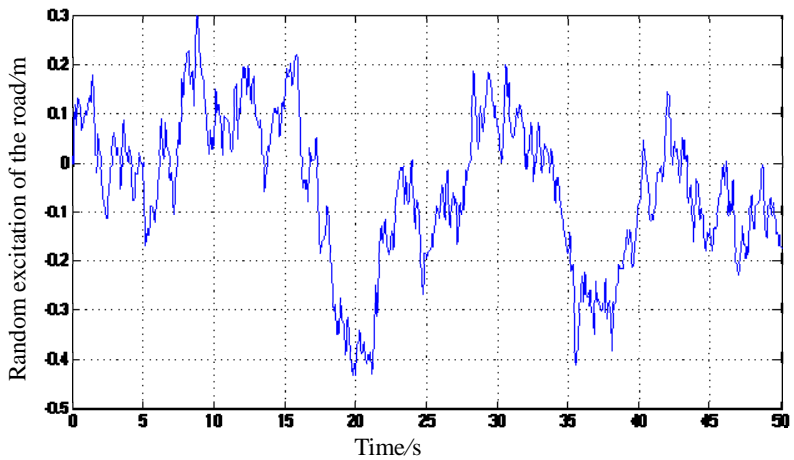

FIGURE VI. THE EXCITATION AT 72KM/H OF THE ROAD WITH SURFACE OF RANK C

In this test, the nominal parameters of hydraulic harvester system for the simulation are $\mathrm{m} 1=40 \mathrm{~kg}, \mathrm{~m} 2=363 \mathrm{~kg}$, $\mathrm{k} 1=182087 \mathrm{~N} / \mathrm{m}, \mathrm{k} 2=20053 \mathrm{~N} / \mathrm{m}, \mathrm{C} 1=200 \mathrm{NS} / \mathrm{m}, \mathrm{C} 2=1388 \mathrm{NS} / \mathrm{m}$ and the input $\mathrm{y} 0$ is the white noise which is shown in Figure VI. And the relative velocity of piston is shown in Figure VII. We can find that the velocity fluctuates greatly which indicates that the output power of the generator changes greatly, as shown in Figure VII.

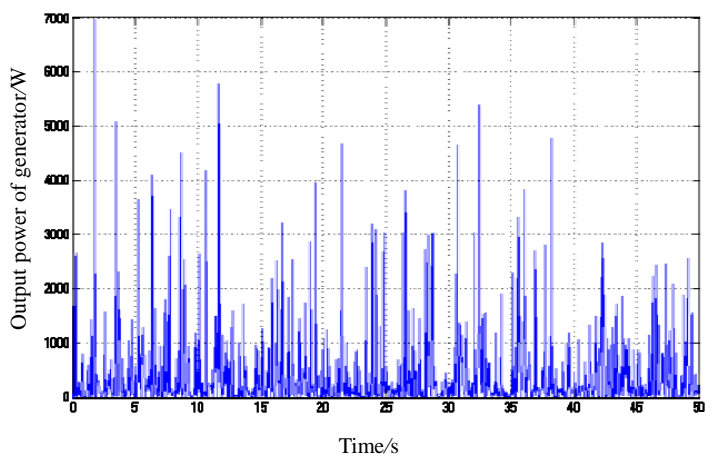

FIGURE VII. THE OUTPUT POWER OF THE GENERATOR

As you can see in Figure VIII, the output power is chaos, so it must be rectified before stored in the battery. Moreover, Figure VIII shows the different output power in different driving condition. When vehicle travelling on the road with surface of rank B at $60 \mathrm{~km} / \mathrm{h}$, the harvester can extracts about $80 \mathrm{~W}$ energy. When vehicle travelling on the road with surface of rank $\mathrm{C}$ at $60 \mathrm{~km} / \mathrm{h}$, the harvester can extracts about $300 \mathrm{~W}$ energy, as shown in Figure VIII. So we can find that the worse condition of road, the more power produced. When the vehicle travels on the different road, it needs different damper force to absorb the vibration. Obviously, the conventional shock absorber can not adapt the different conditions of the road.

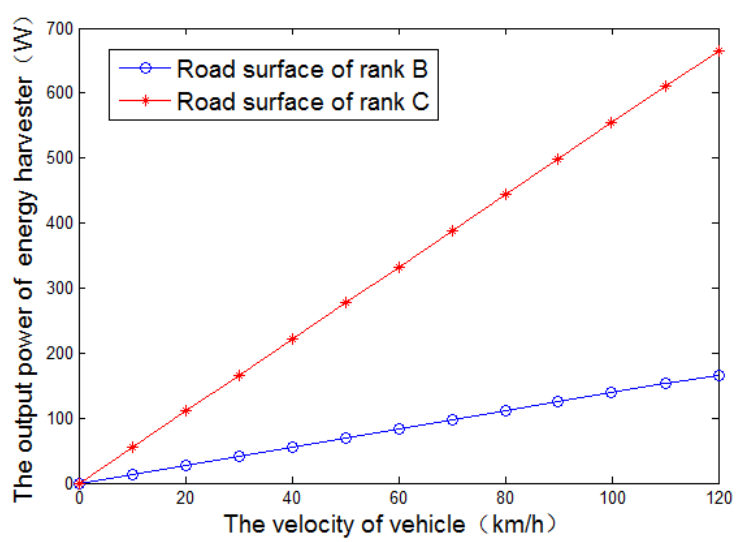

FIGURE VIII. THE OUTPUT POWER OF DIFFERENT VELOCITY AND DIFFERENT ROAD CONDITION

\section{CONCLUSIONS}

Since the conventional shock absorber's damping coefficient is a constant, so it can't adapt different condition of the road. In this paper, we proposed an innovative shock absorber, which based on the hydraulic mechanism. With the hydraulic motor drives the generator, the harvester can extract the vibration energy effectively. And for hydraulic system is easy to be controlled, the harvester's damper coefficient can be changed automatically and adapt the different road condition. The result of simulation indicates that with worse condition of the road, the output power is corresponding higher.

\section{ACKNOWLEDGEMENT}

This work was supported in part by the National Natural Science Foundation of China (Grant No. 51575188), Natural Science Foundation of Guangdong Province (Grant No. 2016A030313492), and Research Foundation of Guangdong Provice (Grant No.2015A020214007).

\section{REFERENCES}

[1] Ruichen Wang, Zhi Chen, Haijun Xu, Karsten Schmidt, Fengshou Gu and Andrew.D.Ball. Modelling and Validation of a Regenerative Shock Absorber System. Proceedings of the 20th International Conference on Automation \& Computing, Cranfield University [C]. Bedfordshire, UK, 12-13 September 2014.

[2] Fodor M, Redfield R. The variable linear transmission for regenerative damp ing in vehicle suspension control [ J ]. Vehicle System Dynamics, 1993, 22 (1) : 1 20.

[3] Noritsugu T. Energy saving of a pneumatic system (2). Energy regenerative control of a pneumatic drive system. Application to active air suspension [J]. Hydraulics \& Pneumatics, 1999, 38 (4) : $1 \sim 4$.

[4] Chuan Li and Peter W Tse. Fabrication and testing of an energyharvesting hydraulic damper [J]. Smart Mater. Struct. 2013.

[5] ChaoChen, Wei-Hsin Liao. A self-sensing magnetorheological damper with power generation [J]. Smart Mater. Struct, 2012.

[6] Bogdan Sapinski. Energy-harvesting linear MR damper: prototyping and testing [J]. Smart Mater.Struct, 2014.

[7] Lei Zuo, Design and characterization of an electromagnetic energy harvester for vehicle suspensions [J]. Smart Materials and Structures, 2010, 19.

[8] Longxin Zhen: Structure and Magnetic Field Analysis of Regenerative Electromagnetic Shock Absorber, WASE International Conference on Information Engineerin, 2010. 
[9] J. H. Beno, D. A. Weeks, D. A. Bresie, A. M. Guenin, J. S. Wisecup, W. Bylsma. Experimental Comparison of Losses for Conventional Passive and Energy Efficient Active Suspension Systems[C]. SAE, 2002.

[10] Weeks D. A., Beno J. H., Guenin A. M., Bresie D. A.. Electromechanical Active Suspension Demonstration for Off-Road Vehicles[C]. SAE, 2000.

[11] Yasuhiro Uchiyama, Masayuki Fujita. Application of Two-Degree-ofFreedom Control to Multi-axis Electrodynamic Shaking System UsingSynthesis and Adaptive Filter [J]. JSME International Journal, c2003, 3:828-835.

[12] Lei Zuo, Energy-harvesting shock absorber with a mechanical motion rectifier [J], Smart Materials and Structures, 2013, 22.

[13] Ryuzo Hayashi, Yoshihiro Suda, Kimihiko Nakano. Anti-Rolling Suspension for an Automobile by Coupled Electromagnetic Devices [J]. Journal of Mechanical Systems for Transportation and Logistics, 2008, Vol. (l): 43-55.

[14] Kimihiko Nakano, Yoshihiro Suda, Shigeyuki Nakadai, Yuji koike. AntiRolling System for Ships with Self-Power Active Control [J]. JSME International Journal, 2001, 3:587-594.

[15] Chew Kuew Wai, Electric Vehicle Energy Harvesting System Regenerative Shock Absorber For Electric Vehicle, IEEE Conference on Sustainable Utilization and Development in Engineering and Technology, 2013. 